\title{
An Excluded-Minor Problem in Matroids
}

\author{
S.R. Kingan ${ }^{1}$ and Manoel Lemos ${ }^{2}$ \\ ${ }^{1}$ Department of Mathematical and Computer Sciences, The Pennsylvania State University \\ Capital College, Middletown, PA 17057-4898, USA \\ srkingan@psu.edu \\ ${ }^{2}$ Departamento de Matematica, Universidade Federal de Pernambuco, Recife, Pernambuco \\ 50740-540, Brazil \\ manoel@dmat.ufpe.br
}

Received May 30, 2003

AMS Subject Classification: 05B35, $05 \mathrm{C} 83$

\begin{abstract}
Consider the class of matroids $M$ with the property that $M$ is not isomorphic to a wheel graph, but has an element $e$ such that both $M \backslash e$ and $M / e$ are isomorphic to a seriesparallel extension of a wheel graph. We give a constructive characterization of such matroids by determining explicitly the 3-connected members of the class. We also relate this problem with excluded minor problems.
\end{abstract}

Keywords: excluded minor, graph, matroid

\section{References}

1. S.R. Kingan, Binary matroids without prisms, prism duals, and cubes, Discrete Math. 152 (1996) 211-224.

2. J.P.S. Kung, Excluding the cycle geometries of the Kuratowski graphs from binary geometries, Proc. London Math. Soc. (3) 55 (1987) 209-242.

3. J.G. Oxley, On nonbinary 3-connected matroids, Trans. Amer. Math. Soc. 300 (1987) 663679.

4. J.G. Oxley, Matroid Theory, Oxford University Press, New York, 1992.

5. P.D. Seymour, Decomposition of regular matroids, J. Combin. Theory Ser. B 28 (1980) 305359. 